\title{
A COOLING FLOW CLUSTER AT REDSHIFT $Z=0.2$
}

\author{
Anna Wolter ${ }^{1}$, I.M. Gioia ${ }^{1,2}$, T. Maccacaro ${ }^{1,2}$, S.L. Morris ${ }^{3}$, R. Nesci' ${ }^{4}$ G.C. Perola ${ }^{4}$, and R. Schild ${ }^{1}$ \\ 1) Harvard/Smithsonian Center for Astrophysics, Cambridge, MA \\ 2) Istituto di Radioastronomia del CNR, Bologna, Italy \\ 3) Mount Wilson and Las Campanas Observatories, Pasadena, CA \\ 4) Istituto Astronomico dell'Universita', Roma, Italy
}

\begin{abstract}
In this paper we discuss the cluster of galaxies 1E0839.9+2938, serendipitously discovered at $X$ ray wavelengths. The collected data imply that this is an example of cooling flow cluster.

\section{Introduction}

Most of the candidates for the study of cooling flows in clusters of galaxies are optically selected clusters: they are typically at low redshifts $(z<0.1)$. To define the onset of the cooling flow phenomenon, it is necessary to include more distant clusters. We show that the $x$-ray selection is a suitable tool to identify candidate cooling flow clusters at higher redshifts. In this paper we present a serendipitous $x$-ray source, $1 E 0839+2938$, identified with a cluster at redshift $z=0.195$. This cluster is a bright $x$-ray emitter (luminosity: $3.9 \times 10^{44} \mathrm{erg} \mathrm{s}^{-1} ; \mathrm{H}_{0}=50 \mathrm{~km} \mathrm{~s}^{-1}$ and $\mathrm{q}_{0}=0$ are adopted throughout the paper.). Its central galaxy is a radio emitter $\left(1.1 \times 10^{24} \mathrm{WHz}^{-1}\right)$, and presents strong optical emission lines. Furthermore, the emission line region of the galaxy (at $\mathrm{H} \alpha$ and [OII]) is not confined to the nucleus, but is extended. While this is only a summary of the major results, a more detailed presentation of the data and interpretation of the results is given in Nesci et al., 1988.
\end{abstract}

\section{The data}

The Extended Medium Sensitivity Survey (EMSS, Gioia et al., 1988) is a complete sample of serendipitous $x$-ray sources, and provides a large sample of high-redshift clusters (up to $z-0.5$ ). One of these sources is found at $08^{\mathrm{h}} 39^{\mathrm{m}} 53.9^{s}+29^{\circ} 38^{\prime} 48^{\prime \prime}$. The source is close to the window supporting structure of the IPC (see Fig.1), and it has a relatively small number of net counts $(-100)$, so an analysis of the $\mathrm{x}$-ray surface brightness distribution is not feasible.

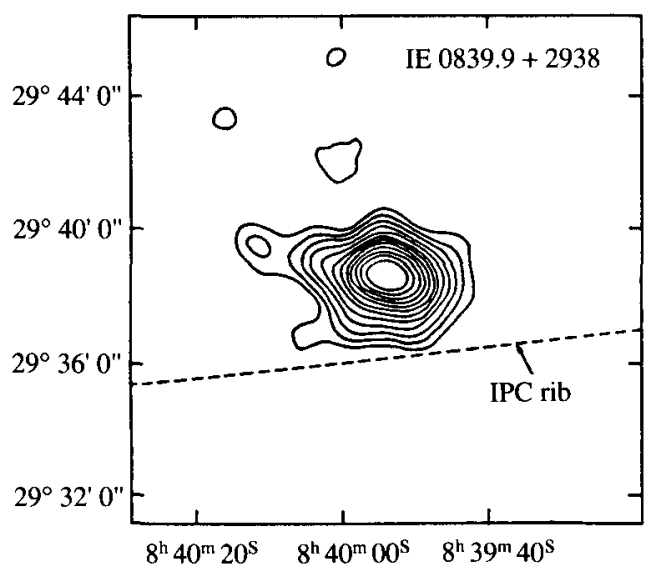

Fig. 1: X-ray contours for 1E $0839+2938$. The first contour corresponds to the $3 \sigma$ level. 
The 0.3-3.5 keV flux is $(2.23 \pm 0.24) \times 10^{-12} \mathrm{erg} \mathrm{cm}^{-2} \mathrm{~s}^{-1}$, computed assuming a Raymond-Smith thermal bremsstrahlung model with a temperature of $6 \mathrm{keV}, 50 \%$ of solar abundance and a hydrogen column density corresponding to the galactic absorption in the direction of the source $\mathbb{N}_{\mathrm{H}}=$ $4.2 \times 10^{20} \mathrm{~cm}^{-2}$; Stark et al., 1989).

A radio source (flux $S=5.6 \mathrm{mJy}$ ) has been detected with the VLA $(6 \mathrm{~cm}, \mathrm{C}$ configuration) at the optical position of the brightest galaxy in the cluster. At the source redshift this flux corresponds to a luminosity of $1.1 \times 10^{24} \mathrm{WHz}^{-1}$, a typical value for compact radio sources in cooling flow clusters (O'Dea and Baum 1986; Jones and Forman 1984).

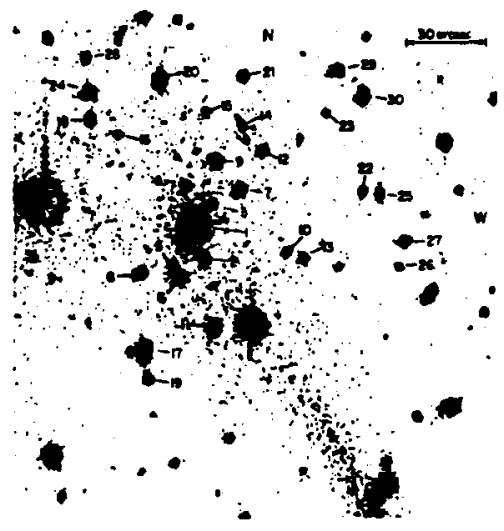

Fig. 2: A CCD image of the field of $1 E 0839.9+2938$, taken with the 24 " telescope at the Whipple Observatory.

Photometry of the cluster was performed with the RCA CCD camera at the Whipple Observatory 24" telescope; a frame is is shown in Fig. 2. Identification spectra and follow-ups for the brightest galaxy, G1, and other cluster members were taken at the Multiple Mirror Telescope with the FOGS (0.42"/pixel along the slit, $4.5 \AA /$ pixel resolution). Absorption features typical for an elliptical galaxy are observed in $\mathrm{Gl}$, as well as $\mathrm{H} \alpha$, [OI], [OII], and [OII] in emission. A redshift of $0.193 \pm 0.001$ is derived. From these long-slit spectra a spatial extent was suggested, but no firm results were obtained because of poor seeing during the observations. Further observations were made with the University of Hawaii $88^{\prime \prime}$ telescope at Mauna Kea $(0.58$ "/pixel along the slit; $26 \AA$ resolution) with two slit orientations (P.A. $0^{\circ}$ and $90^{\circ}$ ). A spectrum is shown in Fig. 3.

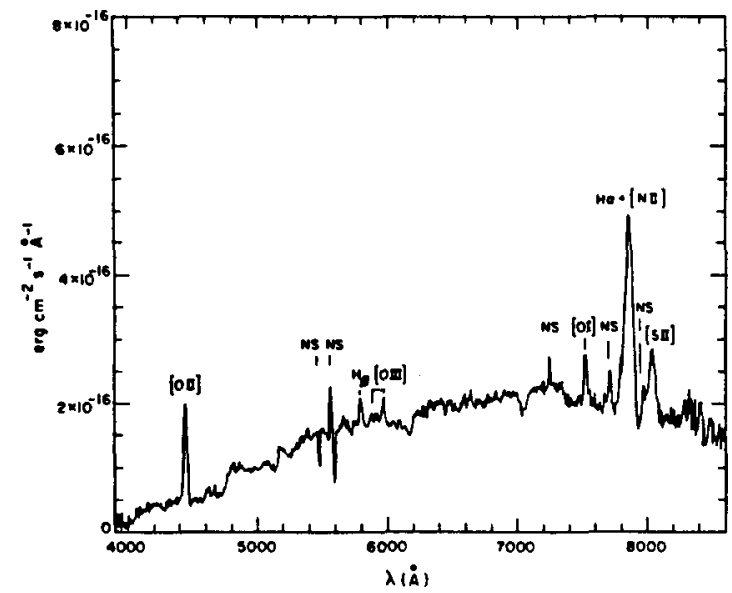

Fig. 3: Optical spectrum, flux calibrated, of galaxy G1 obtained at the University of Hawaii 88" telescope (P.A. $=0^{\circ}$ ) plotted against observed wavelength. 
In Table 1, equivalent widths from the MMT and Hawaii spectra for the stronger emission lines are compared with an average value for $C D$ galaxies in cooling flow $\mathrm{x}$-ray clusters (Hu et al., 1985. Johnstone et al., 1987, Kent and Sargent, 1978). The line ratios observed in galaxy G1 are within the observed range for $\mathrm{CD}$ galaxies with cooling flows. The net line profiles for the strongest lines $(\mathrm{H} \alpha+[\mathrm{NII}]$ and $[\mathrm{OII}])$ are shown in Fig. 4.

Table 1: Equivalent widths, line ratios relative to $\mathrm{H \alpha}$ and typical values for cooling flows.

\begin{tabular}{|c|c|c|c|c|}
\hline Emission line & $\begin{array}{c}\text { E.W.(MMT) } \\
\AA\end{array}$ & $\begin{array}{c}\text { E.W.(UH) } \\
\AA\end{array}$ & $\begin{array}{l}\text { line } \\
\text { ratio }\end{array}$ & $\begin{array}{l}\text { cooling flows } \\
\text { average ratio }\end{array}$ \\
\hline (1) & (2) & (3) & (4) & (5) \\
\hline [OI] $3727 / 29$ & 100. & 93 & 0.53 & $1.5( \pm 1.0)$ \\
\hline $\mathrm{H} \beta$ & 9. & 8. & 0.14 & $0.31( \pm 0.12)$ \\
\hline [OIII]5007 & 9. & 7. & 0.13 & $0.26( \pm 0.12)$ \\
\hline [OI]6300 & 15. & 10. & 0.24 & $0.33( \pm 0.18)$ \\
\hline $\mathrm{H} \alpha$ & 46. $\}$ & & 1.00 & 1.00 \\
\hline [NII]6583 & 71. $\int$ & 114. & 1.54 & $1.43( \pm 0.37)$ \\
\hline [SII]6717/30 & 24. & 20. & 0.47 & $1.02( \pm 0.37)$ \\
\hline
\end{tabular}

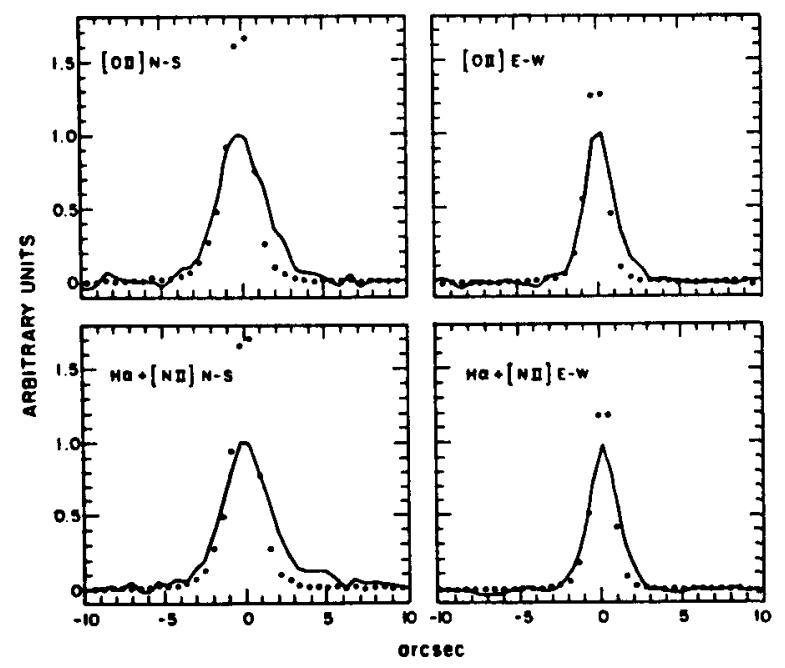

Fig. 4: Spatial profile at $\mathrm{H} \alpha+[\mathrm{NII}]$ and [OII] 3727 at two position angles $\left(0^{\circ}\right.$ and $90^{\circ}$ ). The solid line is the galaxy profile, and the dotted line is a stellar profile for comparison.

The net line profile is obtained by interpolating the galaxy continuum profile at wavelengths redward and blueward of the emission line. The extent of the emission region (at least in the N-S direction) corresponds, at the cluster redshifh, to $13 \mathrm{kpc}$, comparable to the size of A1795 (FWHM-12 kpc; Cowie et al. 1983).

Some relevant parameters for the dominant galaxy are summarized in Table 2. 
Table 2: Relevant data for galaxy G1

$\begin{array}{ll}\text { Total V magnitude } & 16.24 \\ \text { V-R } & 0.82 \\ \text { angle of major axis (from North to East) } & 75^{\circ} \\ \text { eccentricity } & 0.54 \\ \text { Absolute V magnitude } & -24.65 \\ \text { X-ray luminosity }(0.3-3.5 \mathrm{keV}) & 3.9 \times 10^{44} \mathrm{ergs}^{-1} \\ \text { Radio luminosity }(6 \mathrm{~cm}) & 1.1 \times 10^{24} \mathrm{WHz}^{-1}\end{array}$

\section{Conclusions}

Based on the presence of a dominant galaxy at the peak of the $x$-ray emission, the presence of strong emission lines in the optical spectrum of the dominant galaxy, the spatial extent and asymmetry of the line emitting region, and the detection of $\mathrm{Gl}$ at $6 \mathrm{~cm}$, we suggest that 1E0839+2938 is a cooling flow cluster, the second most distant known after 3C295 ( $\mathrm{z}=0.461$, see Henry at al., 1986). Due to the small number of net counts, the $x$-ray data do not allow us to determine the $x$-ray luminosity distribution, and thus the cooling time of the intracluster gas. This study will require future $x$-ray observations, which will be possible with ROSAT and AXAF. A more detailed study of the spatial extent of the optically emitting region will be feasible with the Hubble Space Telescope.

\section{Acknowledgments}

The observations at the UH 88" telescope were obtained in collaboration with Dr. J.P. Henry. This work has received partial financial support from the Smithsonian Scholarly Studies Grant SS88-3-87, and NASA contract NAS8-30751. G.C.P. and R.N. acknowledge financial support from the Italian MPI and CNR/GIFCO.

\section{References}

Cowie, L.b., Hu, E.M., Jenkins, E.B., and York, D.G., 1983, ApJ., 272, 29.

Gioia, I.M., Maccacaro, T., Morris, S.L., Schild, R.E., Stocke, J.T., and Wolter, A., 1988, in "Large scale surveys of the sky", J.J. Condon and J.F. Lockmann eds., in press.

Henry, J. P., and Henricksen, M. J., 1986, Ap. J., 301, 689.

Hu, E.H., Cowie, L.L., Zhong Wang, 1985, Ap.J. Suppl., 59, 447.

Johnstone, R.M., Fabian, A.C., Nulsen, P.EJ., 1987, MNRAS 224, 75.

Jones, C., and Forman, W., 1984, Ap.J. 276, 38

Kent, S.M., Sargent, W.L.W., 1979, Ap.J. 230, 667.

Nesci, R., Gioia, I.M., Maccacaro, T., Morris, S.L., Perola, G.C., Schild, R.E., and Wolter, A., 1988, ApJ., submitted.

O'Dea, C.P., and Baum, S.A., 1986 in Radio Continuum processes in clusters of galaxies, NRAO Workshop n.16, O'Dea and Uson Edts, pag. 141.

Stark, A.A., 1989, in preparation. 